\title{
RESIDUAL POWER SERIES METHOD FOR SOLVING KLEIN-GORDON SCHRÖDINGER EQUATION
}

\author{
Saad A. Manaa ${ }^{\text {a,* }}$, Fadhil H. Easif ${ }^{\text {b }}$ Jomaa J. Murad $^{\text {c }}$ \\ a, b,c Dept. of Mathematics, Faculty of Science, University of Zakho, Kurdistan Region, Iraq - (saad.manaa@uoz.edu.krd , \\ fadhil.easif@uoz.edu.krd, jomaa.murad@staff.uoz.edu.krd)
}

\begin{abstract}
:
In this work, the Residual Power Series Method(RPSM) is used to find the approximate solutions of Klein Gordon Schrödinger (KGS) Equation. Furthermore, to show the accuracy and the efficiency of the presented method, we compare the obtained approximate solution of Klein Gordon Schrödinger equation by Residual Power Series Method(RPSM) numerically and graphically with the exact solution.
\end{abstract}

KEYWORDS: Residual Power Series Method, Klein Gordon Schrödinger.

\section{INTRODUCTION}

The Residual Power Series Method (RPSM) was first presented in 2013 by Abu Arqub [4]. The RPSM is a very significant method for finding the numerical solution of linear and nonlinear differential equations. This method has been applied successfully by many authors to find the numerical solutions for numerous problems, Such as, Alquran, M. (2014) in [2], El-Ajou, A., Arqub, O. A., \& Momani, S. (2015) in [5], Alquran, M. (2015) in [3], and Kumar, S., Kumar, A., \& Baleanu, D. (2016) in [7]. In addition to that, the RPSM has been used by Abu Arqub, O., El-Ajou, A., Bataineh, A. S., \& Hashim, I.(2013) to find the solution of generalized Lane-Emden equations in [1] and it has been applied in 2016 by İnç, M., Korpinar, Z. S., Al Qurashi, M. M., \& Baleanu, D. to find the approximate solutions of some nonlinear equations[6]. It was also applied by Manaa, S. A.,\& Mosa, N. M. in 2019 to solve the KaupBoussinesq System[8] and by Modanli, M., Abdulazeez, S. T., \& Husien, A. M. in 2020 to solve "pseudo hyperbolic partial differential equations with nonlocal conditions"[9]. In this paper, the Residual Power Series Method (RPSM) [1],[6],[8] and [9] is applied to solve Klein-Gordon Schrödinger (KGS) Equation. Moreover, we got all results and figures by using Mathematica program.

\section{MATHEMATICAL MODEL}

Consider the Klein-Gordon Schrödinger (KGS) Equation[10]

$\left\{\begin{array}{c}i q_{t}=-a q_{x x}-b \varphi q \\ \varphi_{t t}=c^{2} \varphi_{x x}-\beta^{2} \varphi+\lambda|q|^{2}\end{array}\right.$

Where $x \in \mathbb{R}, t \geq 0, i=\sqrt{-1}$ and $a, b, c, \beta$ and $\lambda$ are considered to be the arbitrary constants. While, $\boldsymbol{q}$ is the complex nucleon-fields and $\boldsymbol{\varphi}$ is the neutral real mesonfields. Moreover, the system (1) has wide-range applications in many fields such as quantum physics and modern physics [10]-[12].
Equation (1) can be separate into real part and imaginary part. Then, we will get a tripled system (a system of three real equations) as follow:

$\left\{\begin{array}{l}u_{t}=-a v_{x x}-b v \varphi, \\ v_{t}=a u_{x x}+b u \varphi, \\ \varphi_{t t}=c^{2} \varphi_{x x}-\beta^{2} \varphi+\lambda u^{2}+\lambda v^{2} .\end{array}\right.$

For $q=u+i v$.

Where $u$ and $v$ and $\varphi$ are real functions of $x$ and $t$.

\section{BASIC IDEA OF RESIDUAL POWER SERIES METHOD}

Considering the general form of nonlinear partial differential equation:

$L U(x, t)=R[U(x, t)]+N[U(x, t)]$.

Subject to the initial conditions:

$U(x, 0)=f(x)$.

Where $L=\frac{\partial^{m}}{\partial t^{m}}, m \in \mathbb{N}$, is the highest order partial derivative with respect to time $t$. While, the reminder linear term is $R$, the nonlinear operator is $N$.

Now, the solution of Equations (4) by RPSM around the initial point $\mathrm{t}=0$ is written as a power series [1],[6],[8] and [9] as follow:

$$
U(x, t)=\sum_{i=0}^{\infty} f_{i}(x) t^{i} .
$$

Where $i=0,1,2, \ldots$, then, we can definite $U_{n}(x, t)$ to donate the n-th truncated series of $U(x, t)$, i.e.

$$
U_{n}(x, t)=\sum_{i=0}^{n} f_{i}(x) t^{i}
$$

Where $U_{0}=U(x, 0)$ and $f(x)=f_{0}(x)$.

Now, substituting equation (8) into equation (7) we get:

$$
U_{n}(x, t)=U_{0}+\sum_{i=1}^{n} f_{i}(x) t^{i} .
$$

For $t \geq 0, x \in I, n=1,2, \ldots$ 
In order to evaluate the coefficients $f_{i}(x)$, for $i=$ $1,2,3, \ldots, n$ of equation (9), we first defined the residual function for (4), as:

$\operatorname{Res} U(x, t)=L U(x, t)-R[U(x, t)]-N[U(x, t)] \quad(10)$

Then, the $\mathrm{n}$-th residual function $\operatorname{Res}_{n}(x, t)$ is defined as follow:

$$
\begin{gathered}
\operatorname{Res}_{n}(x, t)=L U_{n}(x, t)-R\left[U_{n}(x, t)\right]-N\left[U_{n}(x, t)\right], \\
n=1,2, \ldots
\end{gathered}
$$

As Arqub, O. A. and his colleagues stated in [1], [4] and [5] that:

- $\operatorname{Res} U(x, t)=0$.

- $\lim _{n \rightarrow \infty} \operatorname{Res}_{n}(x, t)=\operatorname{Res} U(x, t), \forall x \in I, t \geq 0$

- $\left.\quad \frac{\partial^{m} \operatorname{Res} U_{n}(x, t)}{\partial t^{m}}\right|_{t=0}=0, m=0,1,2, \ldots, n$.

(12)

Therefore, we can obtain all required coefficients $f_{i}(x)$ (for all $i$ ) of the power series of Equation (4).

\section{DERIVATION OF RPSM FOR SOLVING KGS- SYSTEM}

Assume that equation(2) has the following Initial conditions:

$$
\begin{gathered}
u(x, 0)=u_{0}=f_{0}(x), \\
v(x, 0)=v_{0}=g_{0}(x), \\
\varphi(x, 0)=\varphi_{0}=h_{0}(x), \\
\varphi_{t}(x, 0)=T_{1}(x) .
\end{gathered}
$$

Now, applying RPSM on equation (2) with equation (13). Then, the solution of equation (2) by RPSM around the initial point $\mathrm{t}=0$ is written as:

$$
\begin{aligned}
& u(x, t)=\sum_{i=0}^{\infty} f_{i}(x) t^{i} . \\
& v(x, t)=\sum_{i=0}^{\infty} g_{i}(x) t^{i} . \\
& \varphi(x, t)=\sum_{i=0}^{\infty} h_{i}(x) t^{i} .
\end{aligned}
$$

Where $i=0,1,2, \ldots$, then, we can definite $u_{n}(x, t)$, $v_{n}(x, t)$ and $\varphi_{n}(x, t)$ to give the n-th truncated series of $u(x, t), v(x, t)$ and $\varphi(x, t)$, i.e.

$$
\begin{aligned}
& u_{n}(x, t)=\sum_{i=0}^{n} f_{i}(x) t^{i} . \\
& v_{n}(x, t)=\sum_{i=0}^{n} g_{i}(x) t^{i} . \\
& \varphi_{n}(x, t)=\sum_{i=0}^{n} h_{i}(x) t^{i} .
\end{aligned}
$$

Now, substituting equation (13) into equations (17), (18) and (19) we get:

$$
\begin{aligned}
& u_{n}(x, t)=f_{0}(x)+\sum_{i=1}^{n} f_{i}(x) t^{i} \\
& v_{n}(x, t)=g_{0}(x)+\sum_{i=1}^{n} g_{i}(x) t^{i} \\
& \varphi_{n}(x, t)=h_{0}(x)+T_{1}(x) t+\sum_{i=2}^{n} h_{i}(x) t^{i}
\end{aligned}
$$

In order to calculate the value of coefficients $f_{i}(x), g_{i}(x)$ and $h_{i}(x)$, of equations (20), (21) and (22), for all $i=1,2,3, \ldots, n$. We defined the residual function for equation(2), as:

$\operatorname{Resu}(x, t)=u_{t}+a v_{x x}+b v \varphi$.

$\operatorname{Resv}(x, t)=v_{t}-a u_{x x}-b u \varphi$.

$$
\operatorname{Res} \varphi(x, t)=\varphi_{t t}-c^{2} \varphi_{x x}+\beta^{2} \varphi-\lambda u^{2}-\lambda v^{2} .
$$

Then, the $n$-th residual function $\operatorname{Resu}_{n}(x, t), \operatorname{Res}_{n}(x, t)$ and $\operatorname{Res} \varphi_{n}(x, t)$ is defined as follow:

$$
\begin{aligned}
& \operatorname{Resu}_{n}(x, t)=\frac{\partial u_{n}}{\partial t}+a \frac{\partial^{2} v_{n}}{\partial x^{2}}+b v_{n} \varphi_{n} . \\
& \operatorname{Res}_{n}(x, t)=\frac{\partial v_{n}}{\partial t}-a \frac{\partial^{2} u_{n}}{\partial x^{2}}-b u_{n} \varphi_{n} . \\
& \operatorname{Res}_{n}(x, t)=\frac{\partial^{2} \varphi_{n}}{\partial t^{2}}-c^{2} \frac{\partial^{2} \varphi_{n}}{\partial x^{2}}+\beta^{2} \varphi_{n}-\lambda\left(u_{n}\right)^{2}- \\
& \lambda\left(v_{n}\right)^{2} .
\end{aligned}
$$

For equations (26) and (27) $n=1,2,3, \ldots .$. and for equations (28) $n=2,3, \ldots[9]$. Then, we will have :

- $\operatorname{Resu}(x, t)=0, \operatorname{Resv}(x, t)=0$ and $\operatorname{Res} \varphi(x, t)=0$.

- $\lim _{n \rightarrow \infty} \operatorname{Resu}_{n}(x, t)=\operatorname{Resu}(x, t), \lim _{n \rightarrow \infty} \operatorname{Resv}_{n}(x, t)=$ $\operatorname{Resv}(x, t)$ and

$\lim _{n \rightarrow \infty} \operatorname{Res} \varphi_{n}(x, t)=\operatorname{Res} \varphi(x, t), \forall x \in I, t \geq 0$

$$
\begin{gathered}
\text { - }\left.\frac{\partial^{m} \operatorname{Resu}_{n}(x, t)}{\partial t^{m}}\right|_{t=0}=0,\left.\frac{\partial^{m} \operatorname{Resv}_{n}(x, t)}{\partial t^{m}}\right|_{t=0}=0 \text { and } \\
\left.\frac{\partial^{m} \operatorname{Res} \varphi_{n}(x, t)}{\partial t^{m}}\right|_{t=0}=0, m=0,1,2, \ldots, n .
\end{gathered}
$$

Therefore, to find the solution of equation (2), we need evaluate all required coefficients $f_{i}(x), g_{i}(x)$ and $h_{i}(x)$ (for all $i$ ).

For $n=1$ substitute into equations (20), (21) and (22), then the first RPS approximate solution of equation (2) is :

$$
\begin{gathered}
u_{1}(x, t)=f_{0}(x)+f_{1}(x) t \\
v_{1}(x, t)=g_{0}(x)+g_{1}(x) t
\end{gathered}
$$

Because, in (25) we have $\varphi_{t t}$, then[9],

$$
\varphi_{1}=h_{0}(x)+T_{1}(x) t .
$$

From equations (26), (27) we get:

$$
\begin{aligned}
& \operatorname{Resu}_{1}(x, t)=\frac{\partial u_{1}}{\partial t}+a \frac{\partial^{2} v_{1}}{\partial x^{2}}+b v_{1} \varphi_{1} \\
& \operatorname{Res}_{1}(x, t)=\frac{\partial v_{1}}{\partial t}-a \frac{\partial^{2} u_{1}}{\partial x^{2}}-b u_{1} \varphi_{1}
\end{aligned}
$$

By substituting equations (30) and (31) into equations (33) and (34), we get the $1^{\text {st }}$ residual functions as:

$$
\begin{gathered}
\operatorname{Resu}_{1}(x, t)=f_{1}(x)+a\left(g^{\prime \prime}{ }_{0}(x)+g^{\prime \prime}{ }_{1}(x) t\right)+b\left(g_{0}(x)+\right. \\
\left.g_{1}(x) t\right)\left(h_{0}(x)+h_{1}(x) t\right) . \\
\operatorname{Resv}_{1}(x, t)=g_{1}(x)-a\left(f^{\prime \prime}{ }_{0}(x)+f^{\prime \prime}{ }_{1}(x) t\right)-b\left(f_{0}(x)\right. \\
\left.+f_{1}(x) t\right)\left(h_{0}(x)+h_{1}(x) t\right) .
\end{gathered}
$$

Applying equation (29) on (30) and (31) we have

$$
\begin{aligned}
\left.\operatorname{Resu}_{1}(x, t)\right|_{t=0}= & f_{1}(x)+a\left(g^{\prime \prime}{ }_{0}(x)\right)+b\left(g_{0}(x)\right)\left(h_{0}(x)\right) \\
& =0 \\
\left.\operatorname{Resv}_{1}(x, t)\right|_{t=0}= & g_{1}(x)-a\left(f^{\prime \prime}{ }_{0}(x)\right)-b\left(f_{0}(x)\right)\left(h_{0}(x)\right) \\
& =0
\end{aligned}
$$

Which leads to:

$$
\begin{gathered}
f_{1}(x)=-a\left(g^{\prime \prime}{ }_{0}(x)\right)-b\left(g_{0}(x)\right)\left(h_{0}(x)\right) . \\
g_{1}(x)=a\left(f^{\prime \prime}{ }_{0}(x)\right)+b\left(f_{0}(x)\right)\left(h_{0}(x)\right) .
\end{gathered}
$$

We can rewrite equations (37) and (38) as:

$$
\begin{gathered}
f_{1}(x)=R_{1}(x) . \\
g_{1}(x)=S_{1}(x) .
\end{gathered}
$$


For $R_{1}(x)=-a\left(g^{\prime \prime}{ }_{0}(x)\right)-b\left(g_{0}(x)\right)\left(h_{0}(x)\right)$

and $S_{1}(x)=a\left(f^{\prime \prime}{ }_{0}(x)\right)+b\left(f_{0}(x)\right)\left(h_{0}(x)\right)$

Again, substituting equations (39) and (40) into equations (30) and (31) respectively, with equation (32), we get the first approximate solutions of residual power series method(RPSM) of equation (2):

$$
\begin{gathered}
u_{1}(x, t)=f_{0}(x)+R_{1}(x) t, \\
v_{1}(x, t)=g_{0}(x)+S_{1}(x) t, \\
\varphi_{1}(x, t)=h_{0}(x)+T_{1}(x) t .
\end{gathered}
$$

Setting $n=2$ in equations (20), (21) and (22), then the $2^{\text {nd }}$ RPS approximate solution of equation (2) is:

$$
\begin{gathered}
u_{2}(x, t)=f_{0}(x)+R_{1}(x) t+f_{2}(x) t^{2}, \\
v_{2}(x, t)=g_{0}(x)+S_{1}(x) t+g_{2}(x) t^{2}, \\
\varphi_{2}(x, t)=h_{0}(x)+T_{1}(x) t+h_{2}(x) t^{2} .
\end{gathered}
$$

Then, the $2^{\text {nd }}$ residual functions $\operatorname{Resu}_{2}(x, t)$ , $\operatorname{Resv}_{2}(x, t)$ and $\operatorname{Res} \varphi_{2}(x, t)$ is:

$\operatorname{Resu}_{2}(x, t)=\frac{\partial u_{2}}{\partial t}+a \frac{\partial^{2} v_{2}}{\partial x^{2}}+b v_{2} \varphi_{2}$,

$\operatorname{Resv}_{2}(x, t)=\frac{\partial v_{2}}{\partial t}-a \frac{\partial^{2} u_{2}}{\partial x^{2}}-b u_{2} \varphi_{2}$,

$\operatorname{Res} \varphi_{2}(x, t)=\frac{\partial^{2} \varphi_{2}}{\partial t^{2}}-c^{2} \frac{\partial^{2} \varphi_{2}}{\partial x^{2}}+\beta^{2} \varphi_{2}-\lambda\left(u_{2}\right)^{2}-\lambda\left(v_{2}\right)^{2}$. By substituting equation (42) into equation (43), we get the $2^{\text {st }}$ residual functions as:

$\operatorname{Resu}_{2}(x, t)=R_{1}(x)+2 f_{2}(x) t+a\left(g^{\prime \prime}{ }_{0}(x)+S^{\prime \prime}{ }_{1}(x) t+\right.$ $\left.g^{\prime \prime}{ }_{2}(x) t^{2}\right)+b\left(g_{0}(x)+S_{1}(x) t+g_{2}(x) t^{2}\right)\left(h_{0}(x)+\right.$ $\left.T_{1}(x) t+h_{2}(x) t^{2}\right)$,

$\operatorname{Resv}_{2}(x, t)=S_{1}(x)+2 g_{2}(x) t-a\left(f^{\prime \prime}{ }_{0}(x)+R^{\prime \prime}{ }_{1}(x) t+\right.$ $\left.f^{\prime \prime}{ }_{2}(x) t^{2}\right)-b\left(f_{0}(x)+R_{1}(x) t+f_{2}(x) t^{2}\right)\left(h_{0}(x)+\right.$

$\left.T_{1}(x) t+h_{2}(x) t^{2}\right)$,

$\operatorname{Res} \varphi_{2}(x, t)=2 h_{2}(x)-c^{2}\left(h^{\prime \prime}{ }_{0}(x)+T^{\prime \prime}{ }_{1}(x) t+\right.$ $\left.h^{\prime \prime}{ }_{2}(x) t^{2}\right)+\beta^{2}\left(h_{0}(x)+T_{1}(x) t+h_{2}(x) t^{2}\right)-\lambda\left(f_{0}(x)+\right.$ $\left.R_{1}(x) t+f_{2}(x) t^{2}\right)^{2}-\lambda\left(g_{0}(x)+S_{1}(x) t+g_{2}(x) t^{2}\right)^{2}$.

Applying equation (29) on (44) we get

$$
\begin{array}{r}
\left.\frac{\partial \operatorname{Resu}_{2}(x, t)}{\partial t}\right|_{t=0}=2 f_{2}(x)+a S^{\prime \prime}{ }_{1}(x)+b\left(g_{0}(x) T_{1}(x)+\right. \\
\left.S_{1}(x) h_{0}(x)\right)=0 \\
\left.\frac{\partial \operatorname{Resv}_{2}(x, t)}{\partial t}\right|_{t=0}=2 g_{2}(x)-a R^{\prime \prime}{ }_{1}(x)-b\left(f_{0}(x) T_{1}(x)+\right. \\
\left.R_{1}(x) h_{0}(x)\right)=0
\end{array}
$$

$\left.\operatorname{Res} \varphi_{2}\right|_{t=0}=2 h_{2}(x)-c^{2} h^{\prime \prime}{ }_{0}(x)+\beta^{2} h_{0}(x)$

Which leads to:

$$
-\lambda\left(f_{0}(x)\right)^{2}-\lambda\left(g_{0}(x)\right)^{2}=0
$$

$$
\begin{gathered}
f_{2}(x)=-\frac{1}{2}\left(a{S^{\prime \prime}}_{1}(x)+b\left(g_{0}(x) T_{1}(x)+S_{1}(x) h_{0}(x)\right)\right), \\
g_{2}(x)=\frac{1}{2}\left(a R^{\prime \prime}{ }_{1}(x)+b\left(\begin{array}{c}
f_{0}(x) T_{1}(x)+ \\
R_{1}(x) h_{0}(x)
\end{array}\right)\right), \quad(45) \\
h_{2}(x)=\frac{1}{2}\left(c^{2} h^{\prime \prime}{ }_{0}(x)-\beta^{2} h_{0}(x)+\lambda\left(f_{0}(x)\right)^{2}+\right. \\
\left.\lambda\left(g_{0}(x)\right)^{2}\right) .
\end{gathered}
$$

We can rewrite equations (45) as:

$$
\begin{aligned}
& f_{2}(x)=R_{2}(x), \\
& g_{2}(x)=S_{2}(x), \\
& h_{2}(x)=T_{2}(x) .
\end{aligned}
$$

For

$$
\begin{aligned}
& R_{2}(x)=-\frac{1}{2}\left(a S^{\prime \prime}{ }_{1}(x)+b\left(g_{0}(x) T_{1}(x)+S_{1}(x) h_{0}(x)\right)\right) \\
& S_{2}(x)=\frac{1}{2}\left(a R^{\prime \prime}{ }_{1}(x)+b\left(f_{0}(x) T_{1}(x)+R_{1}(x) h_{0}(x)\right)\right)
\end{aligned}
$$

And

$$
T_{2}(x)=\frac{1}{2}\left(c^{2} h^{\prime \prime}{ }_{0}(x)-\beta^{2} h_{0}(x)+\lambda\left(f_{0}(x)\right)^{2}+\lambda\left(g_{0}(x)\right)^{2}\right)
$$

Again, substituting (46) into (42), we get the $2^{\text {nd }}$ approximate solutions of RPSM of equation (2):

$$
\begin{gathered}
u_{2}(x, t)=f_{0}(x)+R_{1}(x) t+R_{2}(x) t^{2}, \\
v_{2}(x, t)=g_{0}(x)+S_{1}(x) t+S_{2}(x) t^{2}, \\
\varphi_{2}(x, t)=h_{0}(x)+T_{1}(x) t+T_{2}(x) t^{2},
\end{gathered}
$$

And so we can follow the same way for $n=3,4, \cdots$, to find $f_{3}$, $f_{4}, f_{5}, \ldots, g_{3}, g_{4}, g_{5}, \ldots$ and $h_{3}, h_{4}, h_{5}, \ldots$

Then, the approximate solutions of RPSM can take the following forms:

$$
\begin{aligned}
& u(x, t)=\sum_{i=0}^{\infty} f_{i}(x) t^{i} \\
& v(x, t)=\sum_{i=0}^{\infty} g_{i}(x) t^{i} \\
& \varphi(x, t)=\sum_{i=0}^{\infty} h_{i}(x) t^{i} .
\end{aligned}
$$

\section{APPLICATION WITH NUMERICAL RESULTS (TABLES, FIGURES)}

This section will be devoted to find the numerical results (Tables, Figures) of Klein-Gordon Schrödinger (KGS) Equation by using RPSM.

Example: If we take the arbitrary constants of equations (1) and (2) to be: $\left\{b=c=\lambda=\beta=1\right.$ and $\left.a=\frac{1}{2}\right\}$ [11]. Then we get:

$$
\left\{\begin{array}{l}
i q_{t}=-\frac{1}{2} q_{x x}-\varphi q, \\
\varphi_{t t}=\varphi_{x x}-\varphi+|q|^{2} .
\end{array} \quad x \in \mathbb{R}, t \geq 0, \quad i=\sqrt{-1}\right.
$$

and

$\left\{\begin{array}{l}u_{t}=-\frac{1}{2} v_{x x}-v \varphi, \\ v_{t}=\frac{1}{2} u_{x x}+u \varphi, \\ \varphi_{t t}=\varphi_{x x}-\varphi+u^{2}+v^{2}\end{array}\right.$

The exact solitary wave solution of system (49) as in [11],[12] are:

$$
\left\{\begin{array}{c}
q(x, t)=\frac{3 \operatorname{Sech}^{2}\left[\frac{x-x_{0}-t \alpha}{2 \sqrt{1-\alpha^{2}}}\right] e^{i\left(x \alpha+t\left(-\frac{\alpha^{2}}{2}+\frac{1}{2-2 \alpha^{2}}\right)\right.}}{2 \sqrt{2-2 \alpha^{2}}}, \\
\varphi(x, t)=-\frac{3 \operatorname{Sech}^{2}\left[\frac{x-x_{0}-t \alpha}{2 \sqrt{1-\alpha^{2}}}\right]}{4\left(\alpha^{2}-1\right)} .
\end{array}\right.
$$

With the initial conditions:

$$
\left\{\begin{array}{l}
q(x, 0)=\frac{3 \operatorname{Sech}^{2}\left[\frac{x-x_{0}}{2 \sqrt{1-\alpha^{2}}}\right] e^{i x \alpha}}{2 \sqrt{2-2 \alpha^{2}}}, \\
\varphi(x, 0)=-\frac{3 \operatorname{Sech}^{2}\left[\frac{x-x_{0}}{2 \sqrt{1-\alpha^{2}}}\right]}{4\left(\alpha^{2}-1\right)} .
\end{array}\right.
$$

Therefore, the exact solitary wave solutions the system (50) are: 


$$
\left\{\begin{array}{c}
u(x, t)=\frac{3 \operatorname{Sech}^{2}\left[\frac{x-x_{0}-t \alpha}{2 \sqrt{1-\alpha^{2}}}\right] \operatorname{Cos}\left[x \alpha+t\left(-\frac{\alpha^{2}}{2}+\frac{1}{2-2 \alpha^{2}}\right)\right]}{2 \sqrt{2-2 \alpha^{2}}}, \\
v(x, t)=\frac{3 \operatorname{Sech}^{2}\left[\frac{x-x_{0}-t \alpha}{2 \sqrt{1-\alpha^{2}}}\right] \operatorname{Sin}\left[x \alpha+t\left(-\frac{\alpha^{2}}{2}+\frac{1}{2-2 \alpha^{2}}\right)\right]}{2 \sqrt{2-2 \alpha^{2}}}, \\
\varphi(x, t)=-\frac{3 \operatorname{Sech}^{2}\left[\frac{x-x_{0}-t \alpha}{2 \sqrt{1-\alpha^{2}}}\right]}{4\left(\alpha^{2}-1\right)}
\end{array}\right.
$$

\section{With the initial conditions}

$$
\left\{\begin{array}{c}
u(x, 0)=\frac{3 \operatorname{Sech}^{2}\left[\frac{x-x_{0}}{2 \sqrt{1-\alpha^{2}}}\right] \operatorname{Cos}[x \alpha]}{2 \sqrt{2-2 \alpha^{2}}}, \\
v(x, 0)=\frac{3 \operatorname{Sech}^{2}\left[\frac{x-x_{0}}{2 \sqrt{1-\alpha^{2}}}\right] \operatorname{Sin}[x \alpha]}{2 \sqrt{2-2 \alpha^{2}}}, \\
\varphi(x, 0)=-\frac{3 \operatorname{Sech}^{2}\left[\frac{x-x_{0}}{2 \sqrt{1-\alpha^{2}}}\right]}{4\left(\alpha^{2}-1\right)}
\end{array}\right.
$$

Where $x_{0}$ is the initial phase and $|\alpha|>0$ is the propagating velocity of the wave [11].

In the following table and figures we considered initialvalues $\left(\alpha=0.8, x_{0}=-10\right)[11]$.

Table 1. The absolute error between the exact solution and approximate solutions by RPSM of equation (49), $x=10$ and $t \in[0,1]$

\begin{tabular}{|c|c|c|}
\hline Time & $\mid$ qRPSM - qExact $\mid$ & $\mid \varphi$ RPSM $-\varphi$ Exact $\mid$ \\
\hline 0 & 0 & 0 \\
\hline 0.1 & $2.03011067 * 10^{-17}$ & $1.136640964 * 10^{-17}$ \\
\hline 0.2 & $1.67971031 * 10^{-16}$ & $9.410909187 * 10^{-17}$ \\
\hline 0.3 & $5.864410163 * 10^{-16}$ & $3.289471049 * 10^{-16}$ \\
\hline 0.4 & $1.4382761 * 10^{-15}$ & $8.081125316 * 10^{-16}$ \\
\hline 0.5 & $2.907058086 * 10^{-15}$ & $1.636992592 * 10^{-15}$ \\
\hline 0.6 & $5.199395215 * 10^{-15}$ & $2.93600597 \times 10^{-15}$ \\
\hline 0.7 & $8.547065525 * 10^{-15}$ & $4.842746766 * 10^{-15}$ \\
\hline 0.8 & $1.320930239 * 10^{-14}$ & $7.514434237 * 10^{-15}$ \\
\hline 0.9 & $1.947523274 * 10^{-14}$ & $1.113071195 * 10^{-14}$ \\
\hline 1 & $2.766648102 * 10^{-14}$ & $1.589684618 * 10^{-14}$ \\
\hline
\end{tabular}

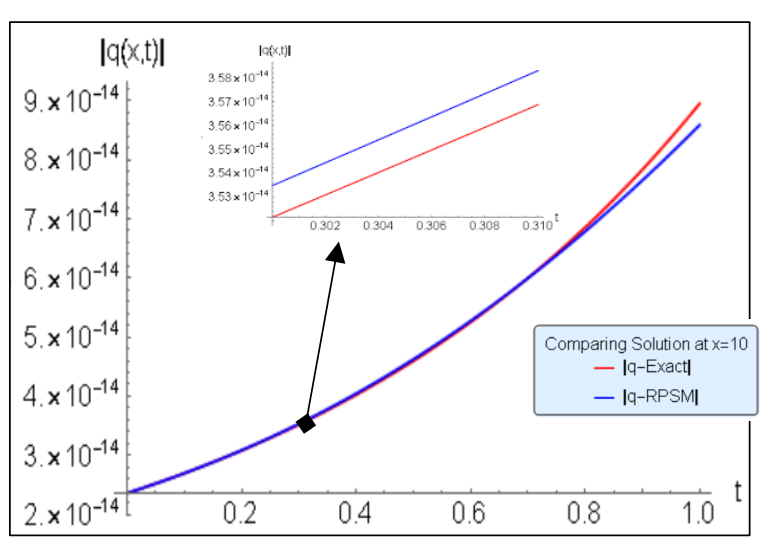

Figure 1: The comparison between RPSM and the exact solution for $|q(x, t)|$, when $x=10$ and $t \in[0,1]$.

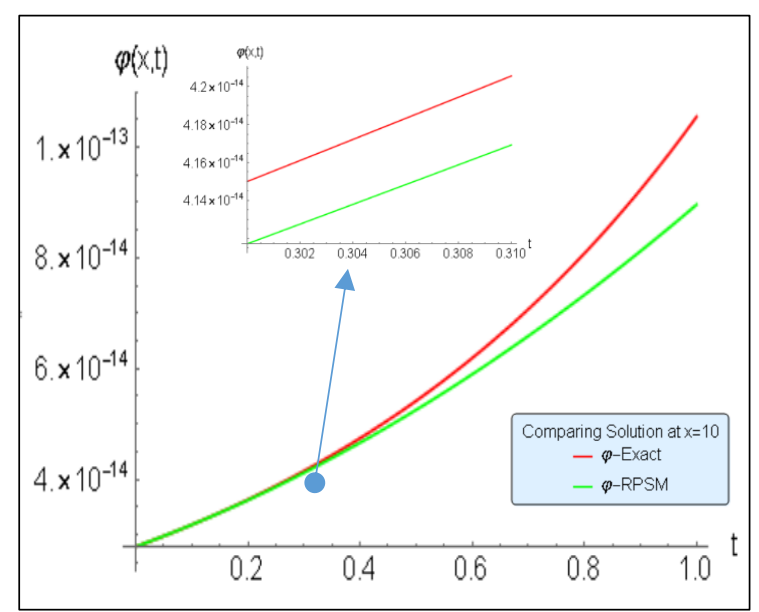

Figure 2: The comparison between RPSM and the exact solution for $\varphi(x, t)$. when $x=10$ and $t \in[0,1]$.

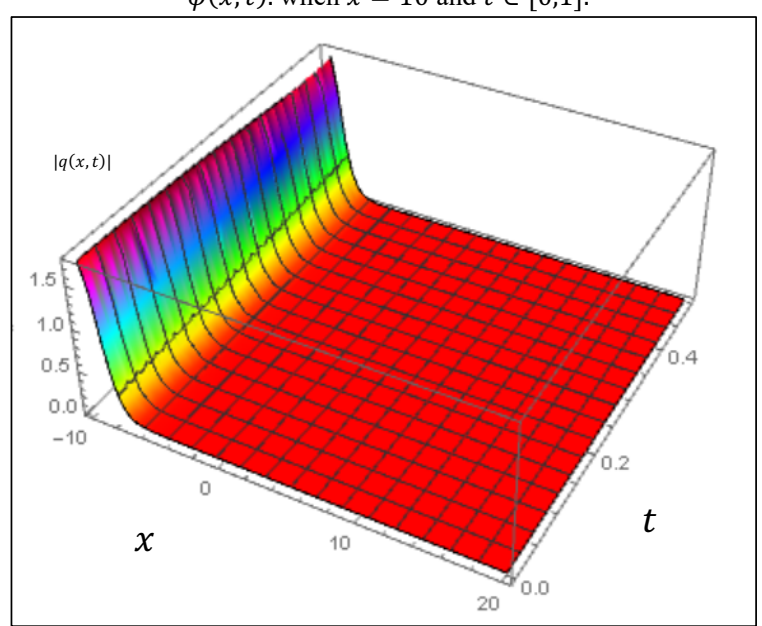

Figure 3:The surfaces of exact solutions $|\boldsymbol{q}(\boldsymbol{x}, \boldsymbol{t})|$, when $x \in[-10,20]$ and $t \in[0,0.5]$.

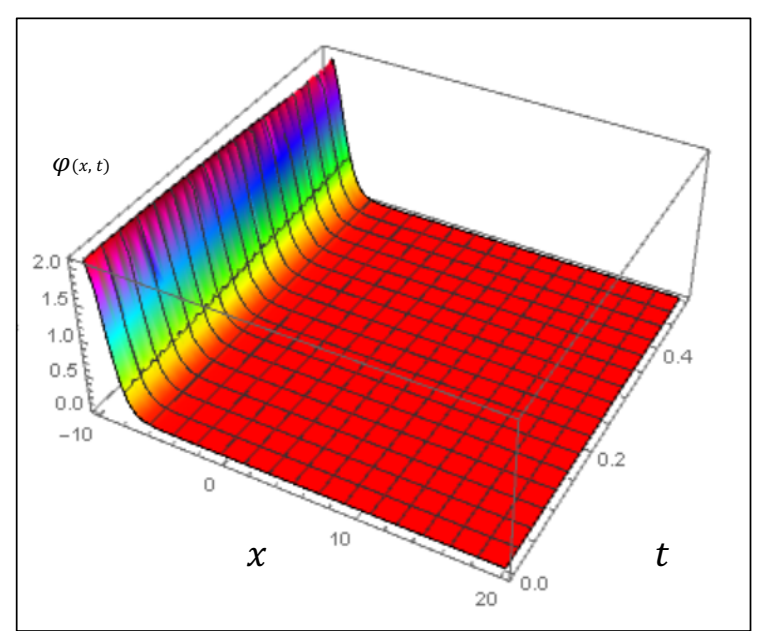

Figure 4:The surfaces of exact solutions $\varphi(x, t)$, when $x \in[-10,20]$ and $t \in[0,0.5]$. 


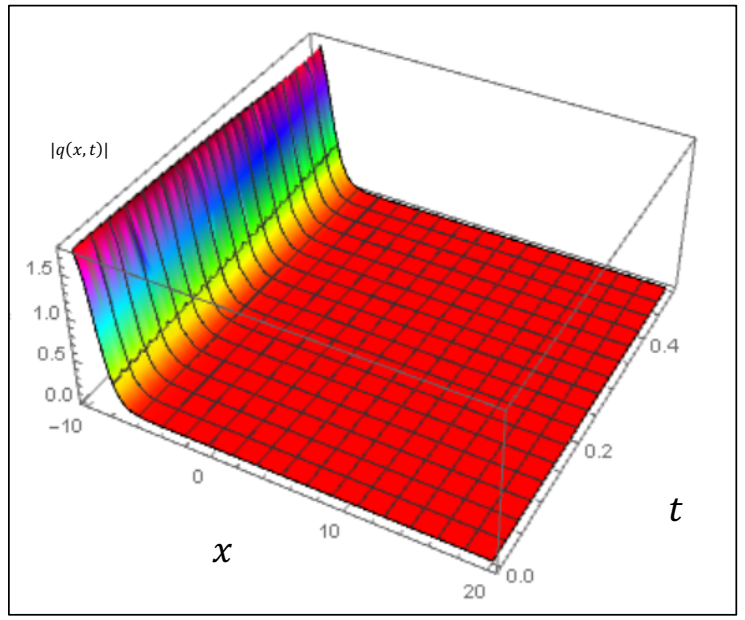

Figure 5: The surfaces of approximate solutions $|q(x, t)|$ by RPSM when $x \in[-10,20]$ and $t \in[0,0.5]$.

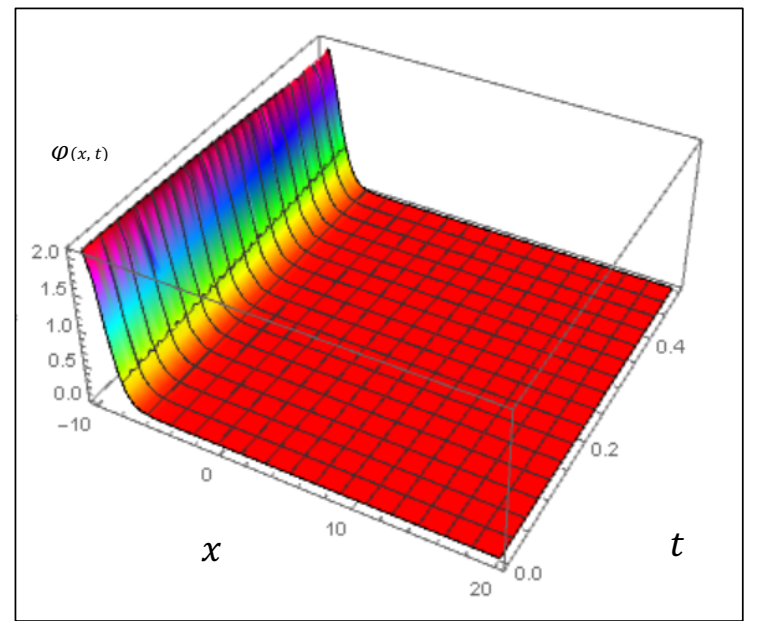

Figure 6: The surfaces of approximate solutions $\varphi(x, t)$ by RPSM when $x \in[-10,20]$ and $t \in[0,0.5]$.

\section{CONCLUSION}

In this paper the Klein-Gordon Schrödinger (KGS) equation was solved numerically by using Residual Power Series Method. We took an example of (KGS) equation to find the comparison between our solution and the exact solution. Moreover, we concluded from Tabl.1 and Figures (1-6) that the RPSM is very accurate and effective in solving (KGS) equation.

\section{REFERENCES}

[1] Abu Arqub, O., El-Ajou, A., Bataineh, A. S., \& Hashim, I. (2013, January). A representation of the exact solution of generalized Lane-Emden equations using a new analytical method. In Abstract and Applied Analysis (Vol. 2013). Hindawi. https://doi.org/10.1155/2013/378593

[2] Alquran, M. (2014). Analytical solutions of fractional foam drainage equation by residual power series method. Mathematical sciences, 8(4), 153-160. https://doi.org/10.1007/s40096-015-0141-1

[3] Alquran, M. (2015). Analytical solution of time-fractional twocomponent evolutionary system of order 2 by residual power series method. J. Appl. Anal. Comput, 5(4), 589-599. https://doi.org/10.11948/2015046

[4] Arqub, O. A. (2013). Series solution of fuzzy differential equations under strongly generalized differentiability. Journal of Advanced Research in Applied Mathematics, 5(1), 31-52. 10.5373/jaram.1447.051912
[5] El-Ajou, A., Arqub, O. A., \& Momani, S. (2015). Approximate analytical solution of the nonlinear fractional KdV-Burgers equation: a new iterative algorithm. Journal of Computational Physics, 293, 81-95. https://doi.org/10.1016/j.jcp.2014.08.004

[6] İnç, M., Korpinar, Z. S., Al Qurashi, M. M., \& Baleanu, D. (2016). A new method for approximate solutions of some nonlinear equations: Residual power series method. Advances in Mechanical Engineering, 8(4), 1687814016644580. https://doi.org/10.1177/1687814016644580

[7] Kumar, S., Kumar, A., \& Baleanu, D. (2016). Two analytical methods for time-fractional nonlinear coupled Boussinesq-Burger's equations arise in propagation of shallow water waves. Nonlinear Dynamics, 85(2), 699-715. https://doi.org/10.1007/s11071-016-2716-2

[8] Manaa, S. A.,\& Mosa, N. M. (2019). Residual Power Series Method for Solving Kaup-Boussinesq System. International Journal of Advanced Trends in Computer Science and Engineering, 8(5), 2089-2095. https://doi.org/10.30534/ijatcse/2019/36852019

[9] Modanli, M., Abdulazeez, S. T., \& Husien, A. M. (2020). A residual power series method for solving pseudo hyperbolic partial differential equations with nonlocal conditions. Numerical Methods for Partial Differential Equations. https://doi.org/10.1002/num.22683.

[10] Tang, X. Y., \& Ding, W. (2007). The general Klein-GordonSchrödinger system: modulational instability and exact solutions. Physica Scripta, 77(1), 015004. https://doi.org/10.1088/00318949/77/01/015004

[11] Wang, J., Dai, H., \& Hui, Y. (2020). Conservative Fourier spectral scheme for higher order Klein-Gordon-Schrödinger equations. Applied Numerical Mathematics, 156, 446-466. https://doi.org/10.1016/j.apnum.2020.05.015.

[12] Zhang, J., \& Kong, L. (2016). New energy-preserving schemes for Klein-Gordon-Schrödinger equations. Applied Mathematical Modelling,40(15-16),6969-6982.

https://doi.org/10.1016/j.apm.2016.02.026 